\title{
Studies of near-barrier fusion induced by neutron-rich nuclei at HRIBF
}

\author{
J. F. Liang ${ }^{1, a}$ \\ Physics Division, Oak Ridge National Laboratory, Oak Ridge, TN 37831, USA
}

\begin{abstract}
Fusion induced by neutron-rich radioactive beams is a topic of current interest. The f ndings will be useful for using radioactive beams to produce superheavy elements. Results from recent measurements performed with neutron-rich radioactive $\mathrm{Sn}$ and Te beams are presented. Coupled-channels calculations were carried out to study the observed sub-barrier fusion enhancement. The fusion probability in Sn on Ni was probed by comparing the evaporation residue cross sections at high excitation energies.
\end{abstract}

\section{Introduction}

Neutron-rich radioactive beams are anticipated to be used for synthesizing new neutron-rich isotopes of superheavy elements[1]. The increase in nuclear size and the presence of a large number of neutron transfer channels with positive Q-values are expected to enhance fusion.

At the Holif eld Radioactive Ion Beam Facility (HRIBF), neutron-rich f ssion fragments can be accelerated to energies above the Coulomb barrier. Because the intensity is several orders lower than that required for heavy element synthesis, the low-intensity beams are used for studying the reaction mechanisms of fusion. The information acquired will be useful for examining the feasibility of using neutron-rich radioactive beams for synthesizing superheavy elements.

The data obtained with radioactive $\mathrm{Sn}$ and Te beams and presented in this paper are preliminary results. The data analysis is still in progress. The f nal results will be published elsewhere.

\section{Near barrier fusion}

The coupled-channels formalism is often used to study sub-barrier fusion enhancement in heavy ion reactions. A distribution of barriers, extracted from the second derivative of the fusion excitation function can reveal the relevant degrees of freedom in the channel coupling[2]. In most reactions, the inelastic excitations of the projectile and target are the dominant channels that contribute to the enhancement whereas the coupling to nucleon transfer plays a minor role. Nevertheless, a few experiments observed a very large sub-barrier fusion enhancement in which a large number of neutron transfer channels with positive Q-values was identif ed to be responsible for the fusion enhancement[3-6].

In reactions involving a neutron-rich radioactive nucleus and a stable nucleus, the number of neutron transfer

\footnotetext{
a e-mail: liangjf@ornl.gov
}

channels and the Q-values can be considerably larger than those in reactions between two stable nuclei. Fusion excitation functions have been measured for neutron-rich Sn and Te nuclei induced reactions to study the inf uence of transfer on fusion.

\subsection{Fusion with ${ }^{40,48} \mathrm{Ca}$}

Several experiments that found large fusion enhancement involved the ${ }^{40} \mathrm{Ca}$ nucleus[3-5].The large sub-barrier fusion enhancement observed in ${ }^{40} \mathrm{Ca}$ on ${ }^{124} \mathrm{Sn}$ is attributed to neutron transfer because the Q-value for transferring 2 to 11 neutrons from ${ }^{124} \mathrm{Sn}$ to ${ }^{40} \mathrm{Ca}$ is positive[4]. There are 14 neutron transfer channels with positive Q-values in ${ }^{132} \mathrm{Sn}+{ }^{40} \mathrm{Ca}$. It is interesting to investigate fusion enhancement in ${ }^{132} \mathrm{Sn}+{ }^{40} \mathrm{Ca}$. Fusion excitation functions for ${ }^{132} \mathrm{Sn}$ on ${ }^{40,48} \mathrm{Ca}$ have been measured in order to compare them with fusion induced by stable ${ }^{124} \mathrm{Sn}$ on the two $\mathrm{Ca}$ isotopes.

Since the compound nucleus formed near the Coulomb barrier primarily decayed by particle evaporation and $\mathrm{fs}$ sion was negligible, only the evaporation residue (ER) cross sections were measured. The targets consisted of molecular forms of calcium, carbon, oxygen, and fuorine on a thin carbon backing $(15 \mu \mathrm{g})$. The target contents and thickness were analyzed by Rutherford back scattering spectrometry. The ERs were identif ed by time-of-fight using timing channel plate detectors and energy loss in the ionization chamber located at zero degrees. The noncalcium contaminants in the target produced ERs with velocities much faster and the atomic number $\mathrm{Z}$ much smaller than the residues of interest. They either missed the trigger of the data acquisition system or could be separated easily by $E-\Delta E$ from the ionization chamber. A detailed description of the apparatus is given in Ref. [7].

The fusion excitation functions for ${ }^{124,132} \mathrm{Sn}$ on ${ }^{40,48} \mathrm{Ca}$ are shown in Fig. 1. The cross section and energy are presented in reduced units to factor out the size and barrier height, respectively, so that it is easier to compare among different reactions. The cross section is divided by 


\section{EPJ Web of Conferences}

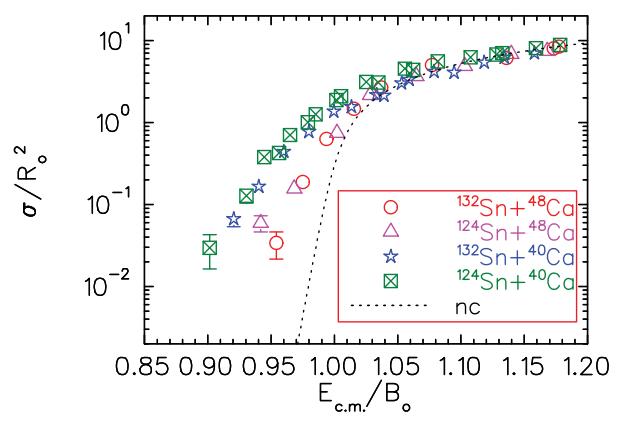

Fig. 1. Fusion excitation function for ${ }^{124,132} \mathrm{Sn}$ on ${ }^{40,48} \mathrm{Ca}$ in reduced units. The nuclear size and the barrier differences among the systems are factored out, $R_{o}^{2}=\left(A_{p}^{1 / 3}+A_{t}^{1 / 3}\right)^{2}$ and $B_{o}=$ $Z_{p} Z_{t} /\left(A_{p}^{1 / 3}+A_{t}^{1 / 3}\right)$ where $Z_{p}\left(Z_{t}\right)$ and $A_{p}\left(A_{t}\right)$ are the atomic number and mass of the projectile (target), respectively. The dotted curve is the prediction of a one-dimensional barrier penetration model (no coupling).

$R_{o}^{2}=\left(A_{p}^{1 / 3}+A_{t}^{1 / 3}\right)^{2}$ and the energy is divided by $B_{o}=$ $Z_{p} Z_{t} /\left(A_{p}^{1 / 3}+A_{t}^{1 / 3}\right)$ where $Z_{p}\left(Z_{t}\right)$ and $A_{p}\left(A_{t}\right)$ are the atomic number and mass of the projectile (target), respectively. The result of a one-dimensional barrier penetration model prediction (no coupling) is shown by the dotted curve. An enhancement of fusion at sub-barrier energies with respect to the uncoupled calculation is observed in all four reactions. The enhancement of fusion with ${ }^{48} \mathrm{Ca}$ is smaller than that with ${ }^{40} \mathrm{Ca}$. The coupled-channels calculation considering inelastic excitation is able to reproduce the excitation function for fusion with ${ }^{48} \mathrm{Ca}$ but signif cantly underpredicts fusion with ${ }^{40} \mathrm{Ca}$. The $\mathrm{Q}$-value for neutron transfer is negative in reactions with ${ }^{48} \mathrm{Ca}$. This suggests that the enhancement in ${ }^{132} \mathrm{Sn}+{ }^{40} \mathrm{Ca}$ can be attributed to neutron transfer. This observation resembles the previous result for ${ }^{40} \mathrm{Ca}+{ }^{124} \mathrm{Sn}[4]$.

As a follow up experiment, we have measured the fusion excitation function of ${ }^{134} \mathrm{Te}$ on ${ }^{40} \mathrm{Ca}$. The ${ }^{134} \mathrm{Te}$ nucleus has the same number of neutrons as ${ }^{132} \mathrm{Sn}$. The reaction has 12 neutron transfer channels with positive Qvalues, 2 channels less than ${ }^{132} \mathrm{Sn}+{ }^{40} \mathrm{Ca}$. As expected, the fusion of ${ }^{134} \mathrm{Te}$ on ${ }^{40} \mathrm{Ca}$ at sub-barrier energies exhibits a large enhancement, similar in magnitude as for ${ }^{132} \mathrm{Sn}+{ }^{40} \mathrm{Ca}$. This enhancement is likely the result of neutron transfer like for the case of ${ }^{132} \mathrm{Sn}+{ }^{40} \mathrm{Ca}$.

\subsection{Fusion with ${ }^{58} \mathrm{Ni}$}

In our previous measurement of fusion of ${ }^{132} \mathrm{Sn}$ and ${ }^{64} \mathrm{Ni}$, it was necessary to include neutron transfer and inelastic excitation in coupled-channels calculations to account for the sub-barrier fusion enhancement. The number of neutron transfer channels that have positive Q-values in ${ }^{132} \mathrm{Sn}+{ }^{58} \mathrm{Ni}$ is three times of that in ${ }^{132} \mathrm{Sn}+{ }^{64} \mathrm{Ni}$ and comparable to that in ${ }^{132} \mathrm{Sn}+{ }^{40} \mathrm{Ca}$. With this in mind, we have measured the fusion excitation function for ${ }^{132} \mathrm{Sn}+{ }^{58} \mathrm{Ni}$ to explore whether transfer will enhance fusion the same way as in ${ }^{132} \mathrm{Sn}+{ }^{40} \mathrm{Ca}$.

In ${ }^{132} \mathrm{Sn}+{ }^{58} \mathrm{Ni}$, $\mathrm{f}$ ssion and $\mathrm{ER}$ cross sections were measured. The ERs were identif ed by the time-of-f ight and

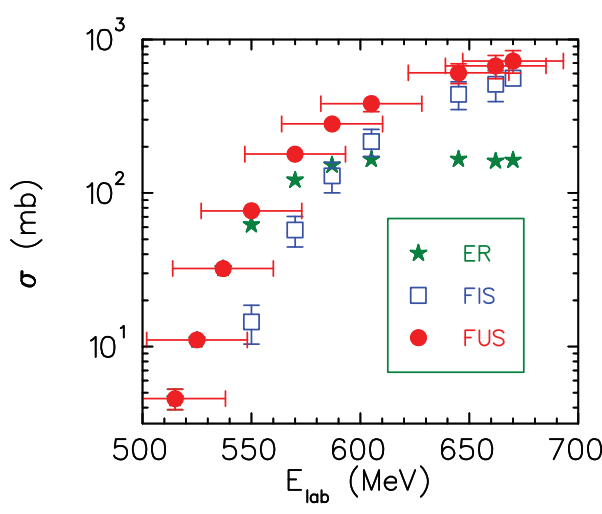

Fig. 2. Fusion excitation function for ${ }^{132} \mathrm{Sn}$ on ${ }^{58} \mathrm{Ni}$. The fusion cross sections are shown by the solid circles, f ssion by the open squares, and ER by the solid stars. The horizontal bars on the fusion data points refer to the energy loss of the beam in the target.

energy loss using the methods described in Ref. [7]. The $\mathrm{f}$ ssion fragments were detected by a double-sided annular silicon strip detector. A description of the analysis of f ssion events can be found in Ref. [8]. The fusion excitation function is displayed in Fig. 2. The fusion cross sections, the sum of $f$ ssion and ER cross sections, are shown by the solid circles, f ssion by the open squares, and ER by the solid stars. At energies below $550 \mathrm{MeV}$, f ssion is negligible and therefore the ER cross section is taken as the fusion cross section. The horizontal bars on the fusion data points refer to the energy loss of the beam in the target. The data points are plotted for reaction energies calculated at the middle of the target. A thick-target yield cross section method was used to deduce the effective reaction energies because of the $1 \mathrm{mg} / \mathrm{cm}^{2}$ target used[8].

In Fig. 3, the fusion excitation function for ${ }^{132} \mathrm{Sn}+{ }^{58} \mathrm{Ni}$ is plotted along with those for ${ }^{132} \mathrm{Sn}+{ }^{64} \mathrm{Ni}$, and ${ }^{124} \mathrm{Sn}+{ }^{58,64} \mathrm{Ni}$. When the nuclear size and barrier height are factored out, all the excitation functions overlap. In contrast, in the fusion of $\mathrm{Sn}$ and $\mathrm{Ca}$ the reactions with the neutron def cient ${ }^{40} \mathrm{Ca}$ show a larger sub-barrier enhancement, as displayed in Fig. 1. The coupled-channels calculation including inelastic excitation for ${ }^{132} \mathrm{Sn}+{ }^{58} \mathrm{Ni}$ is shown by the solid curve and the uncoupled barrier penetration calculation by the dotted curve. It can be seen that the sub-barrier enhancement for ${ }^{132} \mathrm{Sn}+{ }^{58} \mathrm{Ni}$ due to neutron transfer is small unlike that observed in ${ }^{132} \mathrm{Sn}+{ }^{40} \mathrm{Ca}$. Possible explanations for the difference between ${ }^{132} \mathrm{Sn}+{ }^{58} \mathrm{Ni}$ and ${ }^{132} \mathrm{Sn}+{ }^{40} \mathrm{Ca}$ will be discussed in the next section.

\subsection{Coupled-channels analysis}

Barrier distributions extracted from the double differentiation of the fusion excitation function are very useful for studying the relevant reactions in enhancing sub-barrier fusion. It is necessary to measure the fusion cross section to high precision and at small energy intervals. The intensity of radioactive beams produced at present is too low to be practical for such measurements. In order to understand 


\section{FUSION11}

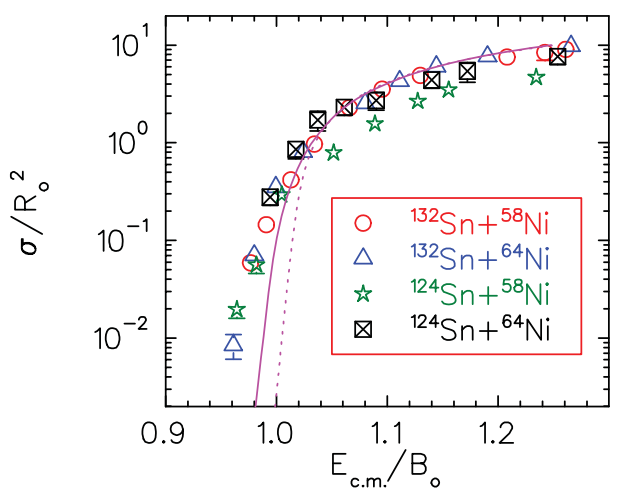

Fig. 3. Reduced fusion excitation functions for ${ }^{132} \mathrm{Sn}+{ }^{58} \mathrm{Ni}$ (circles), ${ }^{132} \mathrm{Sn}+{ }^{64} \mathrm{Ni}$ (triangles), ${ }^{124} \mathrm{Sn}+{ }^{58} \mathrm{Ni}$ (stars), and ${ }^{124} \mathrm{Sn}+{ }^{64} \mathrm{Ni}$ (squares). The coupled-channels calculation including inelastic excitation for ${ }^{132} \mathrm{Sn}+{ }^{58} \mathrm{Ni}$ is shown by the solid curve and the uncoupled barrier penetration calculation by the dotted curve.

Table 1. Reactions and the number of neutron transfer channels that have positive Q-values.

\begin{tabular}{ccccc}
\hline reaction & ${ }^{32} \mathrm{~S}+{ }^{96} \mathrm{Zr}$ & ${ }^{40} \mathrm{Ca}+{ }^{96} \mathrm{Zr}$ & ${ }^{40} \mathrm{Ca}+{ }^{124} \mathrm{Sn}$ & ${ }^{58} \mathrm{Ni}+{ }^{124} \mathrm{Sn}$ \\
neut. trans. & 6 & 9 & 10 & 11 \\
\hline
\end{tabular}

the differences in fusion influence by neutron transfer in ${ }^{40} \mathrm{Ca}$ and ${ }^{58} \mathrm{Ni}$, we can study and compare stable beam data. Table 1 lists the number of neutron transfer channels with positive Q-values for some reactions. Among these reactions there are pairs that have a common projectile or target. A large sub-barrier fusion enhancement owing to neutron transfer has been observed in ${ }^{32} \mathrm{~S}+{ }^{96} \mathrm{Zr}[6]$, ${ }^{40} \mathrm{Ca}+{ }^{96} \mathrm{Zr}[3]$, and ${ }^{40} \mathrm{Ca}+{ }^{124} \mathrm{Sn}[4]$ but little enhancement was seen in ${ }^{58} \mathrm{Ni}+{ }^{124} \mathrm{Sn}[9]$. The fusion excitation functions for the firs three reactions have been measured to high precision. Coupled-channels calculations have been carried out to examine the barrier distributions and compare with the experimental data. The objective is to identify the contributing factors that make the effect of neutron transfer stand out. Furthermore, comparing ${ }^{40} \mathrm{Ca}$ and ${ }^{58} \mathrm{Ni}$ on ${ }^{124} \mathrm{Sn}$ may shed some light on the radioactive beam measurements, ${ }^{132} \mathrm{Sn}$ on ${ }^{40} \mathrm{Ca}$ and ${ }^{58} \mathrm{Ni}$.

This coupled-channels analysis uses a version of CCMOD [10] which can handle multiphonon excitation. Although this code uses some approximations, it produces results comparable to the exact code CCFULL[11]. The main reason for adopting this code is its fl xibility where individual channels and the coupling schemes can be manipulated by hand. This is useful for performing exploratory studies. The nuclear structure properties for inelastic excitation are taken from literature[12,13]. The interaction potentials are obtained from systematics[14].

The coupled-channels calculations considering inelastic excitation of the lowest quadrupole $\left(2^{+}\right)$and octupole $\left(3^{-}\right)$states of the projectile and target, double phonon states $\left(\left(2^{+}\right)^{2},\left(3^{-}\right)^{2}, 2^{+} \otimes 3^{-}\right)$, and mutual excitation of projectile and target up to $7 \mathrm{MeV}$ are shown in Figure 4. The upper panel displays the predicted excitation functions and the lower panel the barrier distributions. At energies be-
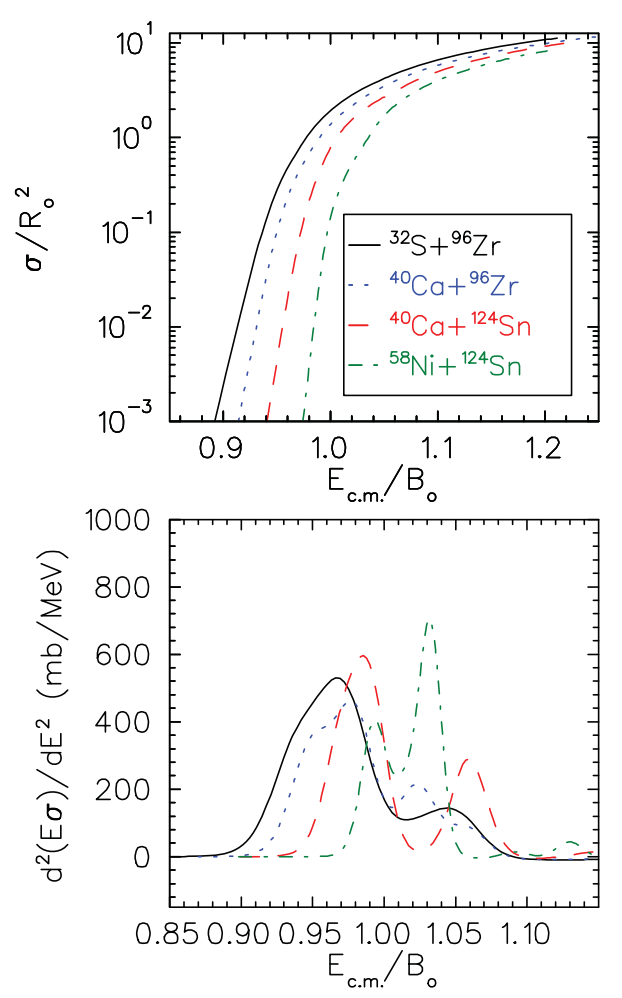

Fig. 4. Upper panel: Fusion excitation functions obtained from coupled-channels calculations considering inelastic excitation for ${ }^{32} \mathrm{~S}+{ }^{96} \mathrm{Zr}$ (solid curve), ${ }^{40} \mathrm{Ca}+{ }^{96} \mathrm{Zr}$ (dotted curve), ${ }^{40} \mathrm{Ca}+{ }^{124} \mathrm{Sn}$ (dashed curve), and ${ }^{58} \mathrm{Ni}+{ }^{124} \mathrm{Sn}$ (dash-dotted curve). Lower panel: Barrier distributions extracted from the calculated excitation functions.

low the barrier, ${ }^{32} \mathrm{~S}+{ }^{96} \mathrm{Zr}$ has the largest cross section, followed by ${ }^{40} \mathrm{Ca}+{ }^{96} \mathrm{Zr},{ }^{40} \mathrm{Ca}+{ }^{124} \mathrm{Sn}$, and ${ }^{58} \mathrm{Ni}+{ }^{124} \mathrm{Sn}$. This reproduces the trend of the experimental data. It is noticed that the centroid of the low-energy barrier for ${ }^{58} \mathrm{Ni}+{ }^{124} \mathrm{Sn}$ is higher and the width is narrower than the other three reactions. It is necessary to include transfer to reproduce the fusion excitation function. To simplify the calculation, only one transfer channel is considered. The Q-value for two-neutron transfer is used and the coupling constant is adjusted to fi the excitation function and barrier distribution. Shown in Fig. 5 are the excitation functions and barrier distributions for ${ }^{40} \mathrm{Ca}+{ }^{124} \mathrm{Sn}$. The predicted excitation function taking into account inelastic excitation and transfer is in fair agreement with the data as shown by the dashed curve. The low-energy barrier from coupling to the inelastic excitation splits into two. This reproduces qualitatively the feature of the experimental barrier distribution. A similar calculation is done for the ${ }^{58} \mathrm{Ni}+{ }^{124} \mathrm{Sn}$ reaction. The barrier distribution is shown in the lower panel of Fig 6. As can be seen, the low-energy barrier is pushed to lower energy but not as significantl as for ${ }^{40} \mathrm{Ca}+{ }^{124} \mathrm{Sn}$. The fusion excitation function is not suitable for extracting the barrier distribution. It is desirable to have such data available for comparison. Calculations for the other two reactions, ${ }^{32} \mathrm{~S}+{ }^{96} \mathrm{Zr}$ and ${ }^{40} \mathrm{Ca}+{ }^{96} \mathrm{Zr}$, reproduce the excitation function and barrier distribution fairly well. 


\section{EPJ Web of Conferences}
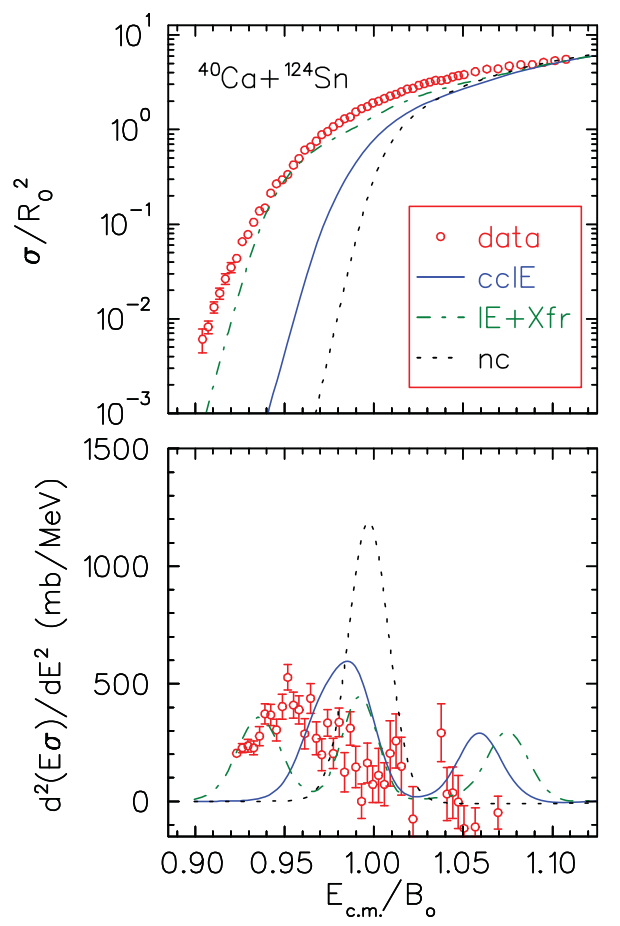

Fig. 5. Upper panel: Fusion excitation functions for ${ }^{40} \mathrm{Ca}+{ }^{124} \mathrm{Sn}$. The data are shown by the open circles[4]. The solid curve is for the coupled-channels calculation including inelastic excitation, the dashed-dotted curve for the calculation including inelastic excitation and transfer, and the dotted curve for the uncoupled calculation. Lower panel: Barrier distributions.

One of the prominent differences in the barrier distribution between ${ }^{40} \mathrm{Ca}+{ }^{124} \mathrm{Sn}$ and ${ }^{58} \mathrm{Ni}+{ }^{124} \mathrm{Sn}$ is the position of the low-energy barrier. Because ${ }^{40} \mathrm{Ca}+{ }^{124} \mathrm{Sn}$ and ${ }^{58} \mathrm{Ni}+{ }^{124} \mathrm{Sn}$ share the same target, the barrier distribution originated from coupling to the target excitations is expected to be similar. Figure 7 compares the coupling to the lowest $2^{+}$and $3^{-}$states. Because the $3^{-}$state is very collective in ${ }^{40} \mathrm{Ca}$, coupling to this state produces a low-energy barrier with the centroid much lower than ${ }^{58} \mathrm{Ni}$. Although the contribution of channel couplings is not necessarily a coherent sum, it is conceivable that this low-energy barrier overlaps with the barrier produced by coupling to transfer. As a matter of fact, the coupled-channels calculation for coupling to transfer using the Q-value for two-neutron transfer and the coupling constant that reproduces the excitation function, produces a low-energy barrier located at $\mathrm{E}_{\text {c.m. }} / \mathrm{B}_{o}=0.95$. This barrier overlaps with that from coupling to the $3^{-}$state of ${ }^{40} \mathrm{Ca}$ which may explain the pronounced effect of coupling to transfer observed with ${ }^{40} \mathrm{Ca}$. The role of coupling to high-lying $3^{-}$states has been discussed in Ref. $[15,16]$.

\section{Fusion probability}

Fusion hindrance in heavy systems is attributed to quasi$\mathrm{f}$ ssion where the reacting nuclei surpass the Coulomb barrier but fail to reach inside the saddle point, and reseparate
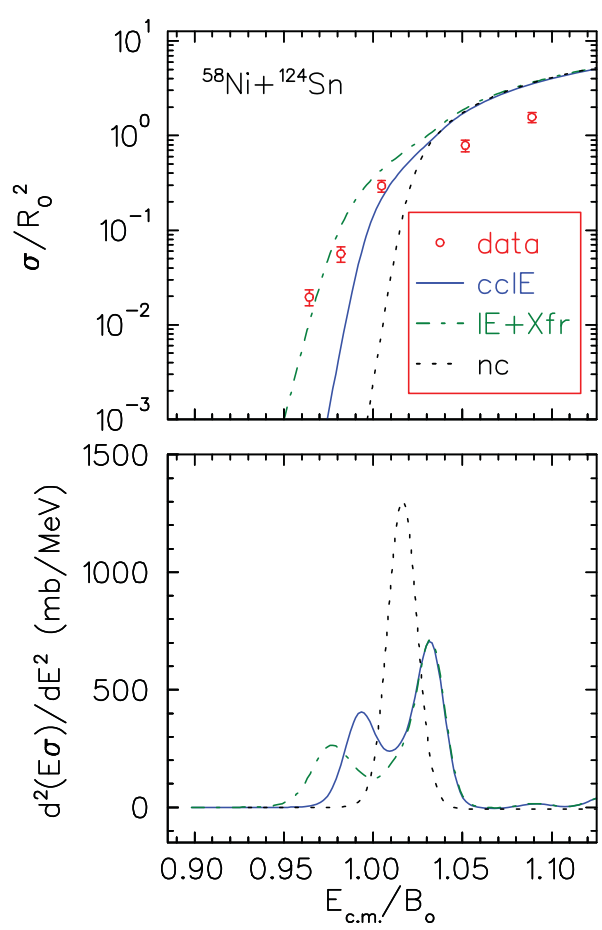

Fig. 6. Upper panel: Fusion excitation functions for ${ }^{58} \mathrm{Ni}+{ }^{124} \mathrm{Sn}$. The data are shown by the open circles[9]. The solid curve is for the coupled-channels calculation including inelastic excitation, the dashed-dotted curve for the calculation including inelastic excitation and transfer, and the dotted curve for the uncoupled calculation. Lower panel: Barrier distributions.

into large mass fragments. It is important to get a better understanding of this process in order to reliably predict the production cross section for superheavy elements. In particular, the role played by neutron excess in affecting fusion hindrance is not well understood. Two theoretical models give conf icting results[17].

The ER cross section $\left(\sigma_{E R}\right)$ can be parametrized as the product of the capture cross section $\left(\sigma_{c a p}\right)$, fusion probability $\left(P_{C N}\right)$, and survival probability $\left(W_{\text {sur }}\right)$,

$$
\sigma_{E R}=\sigma_{c a p} P_{C N} W_{\text {sur }} .
$$

The angular momentum averaged fusion probability is proportional to the ER cross section factoring out the entrance channel kinematics, $\pi \lambda^{2}$, where $1 / \lambda=k=\sqrt{2 \mu E} / \hbar, \mu$ the reduced mass of the projectile and target, and $E$ the centerof-mass energy[18].

$$
<P_{C N}(E)>_{l}=\frac{\sigma_{E R}}{\pi \lambda^{2} \Sigma(2 l+1) T_{l}(E) W_{s u r}(E, l)}
$$

When fusion reactions occur at high excitation energies, the transmission coefficient, $T_{l}(E)$, becomes 1 . For reactions producing the same compound nucleus, the survival probability is the same provided the excitation energy and the angular momentum are the same. At sufficiently high excitation energies, it is possible to match the spin distribution of different entrance channels for the ERs. This can be achieved by populating the spin distribution well above 


\section{FUSION11}
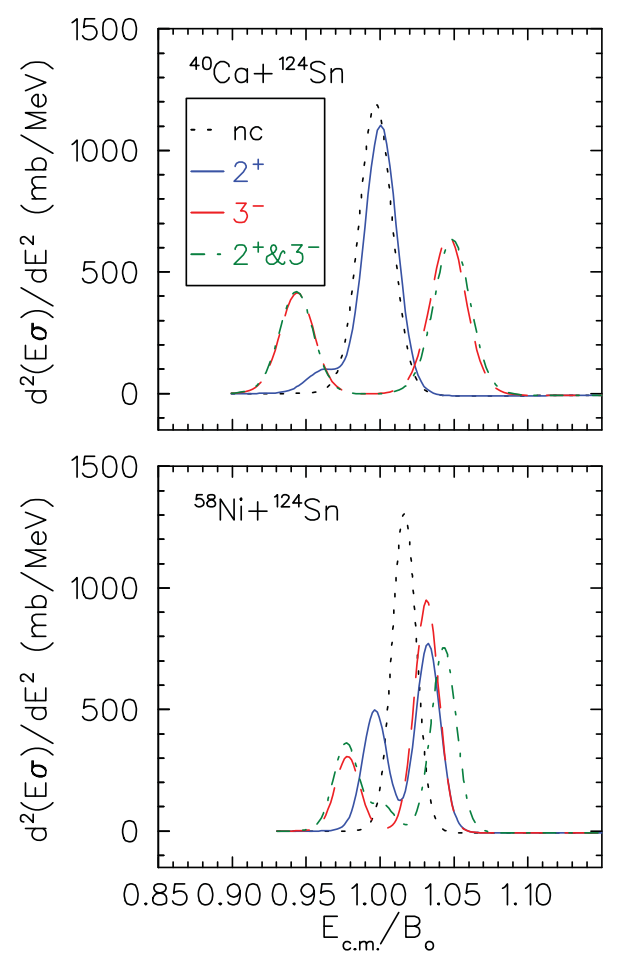

Fig. 7. Barrier distributions calculated by coupling to the lowest $2^{+}$(solid curves), $3^{-}$(dashed curves), and $2^{+}$and $3^{-}$(dasheddotted curve) states for ${ }^{40} \mathrm{Ca}+{ }^{124} \mathrm{Sn}$ (upper panel) and ${ }^{58} \mathrm{Ni}+{ }^{124} \mathrm{Sn}$ (lower panel). The uncoupled calculation is shown by the dotted curves.

the angular momentum for which the fissio barrier diminishes. Therefore, one can study the fusion probability of a dinuclear system evolving into a compound nucleus by comparing ER cross sections at high energies[19,20]. We have measured the ER cross sections for ${ }^{127} \mathrm{Sn}+{ }^{64} \mathrm{Ni}$ and ${ }^{132} \mathrm{Sn}+{ }^{58} \mathrm{Ni}$ to study the isotopic and entrance-channel mass-asymmetry dependence of the fusion probability.

A comparison of the reduced ER cross sections, $\sigma_{E R} / \pi \lambda^{2}$, as a function of excitation energy is presented in Fig. 8. The plan was to measure the ER cross sections for ${ }^{126} \mathrm{Sn}+{ }^{64} \mathrm{Ni}$ and compare with those for ${ }^{132} \mathrm{Sn}+{ }^{58} \mathrm{Ni}$ because the two reactions produce the same compound nucleus. Due to a technical issue, the ${ }^{126} \mathrm{Sn}+{ }^{64} \mathrm{Ni}$ reaction will be measured in a later time.

The ${ }^{127} \mathrm{Sn}+{ }^{64} \mathrm{Ni}$ reaction produces a compound nucleus with one more neutron than ${ }^{126} \mathrm{Sn}+{ }^{64} \mathrm{Ni}$ and ${ }^{132} \mathrm{Sn}+{ }^{58} \mathrm{Ni}$. The survival probability is slightly higher because of this extra neutron. In order to make a comparison, the survival probability is calculated at the same excitation energy for the two compound nuclei and is used to normalize the reduced ER cross sections for ${ }^{127} \mathrm{Sn}+{ }^{64} \mathrm{Ni}$. The normalized result is shown by the fille circles in Fig. 8. At excitation energies greater than $90 \mathrm{MeV}$, the reduced ER cross sections for ${ }^{132} \mathrm{Sn}+{ }^{58} \mathrm{Ni}$ and normalized ${ }^{127} \mathrm{Sn}+{ }^{64} \mathrm{Ni}$ are consistent within uncertainty. Thus, it is concluded that the fusion probability is the same for both reactions. In contrast, the fusion probability studied for ${ }^{90,92,94,96} \mathrm{Zr}$ on ${ }^{124} \mathrm{Sn}$ which produce heavier compound nuclei decreases as the number of neutrons increases in the compound nu-

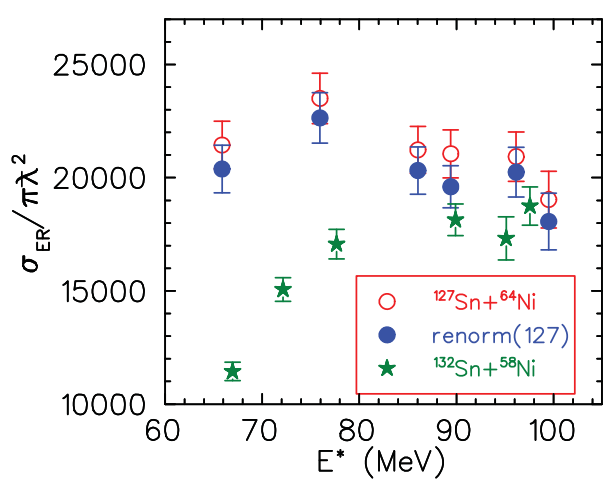

Fig. 8. Angular momentum averaged fusion probability, $\sigma_{E R} / \pi \lambda^{2}$ as a function of excitation energy. The ${ }^{132} \mathrm{Sn}+{ }^{58} \mathrm{Ni}$ is shown by the fille stars, and the ${ }^{127} \mathrm{Sn}+{ }^{64} \mathrm{Ni}$ by open circles. The fille circles are for ${ }^{127} \mathrm{Sn}+{ }^{64} \mathrm{Ni}$ normalized by the survival probability of ${ }^{126}+{ }^{64} \mathrm{Ni}$.

cleus. It appears that more measurements for heavier systems are needed to help understand the fusion probability. In addition, fusion hindrance has been reported in stable $\mathrm{Sn}$ and Ni fusion[9]. A comparison with light ion induced reactions that produce the same compound nucleus as ${ }^{132} \mathrm{Sn}+{ }^{58} \mathrm{Ni}$ where no hindrance is assumed, will be required to see whether fusion hindrance is present in radioactive $\mathrm{Sn}$ induced fusion.

\section{Summary}

Fusion induced by radioactive $\mathrm{Sn}$ beams on $\mathrm{Ca}$ and $\mathrm{Ni}$ targets has been measured. A large sub-barrier fusion enhancement attributed to neutron transfer is observed in the reactions with ${ }^{40} \mathrm{Ca}$. The coupled-channels analysis shows that coupling to the lowest $3^{-}$state of ${ }^{40} \mathrm{Ca}$ makes the effect of neutron transfer more pronounced because of the overlap with the barrier originated from coupling to transfer.

The fusion probability for ${ }^{127} \mathrm{Sn}+{ }^{64} \mathrm{Ni}$ and ${ }^{132}+{ }^{58} \mathrm{Ni}$ has been compared. Within experimental uncertainty and corrected for the survival probability, the fusion probability for the two reactions with a different entrance massasymmetry is in agreement. Future measurements will study heavier compound nuclei made in neutron-rich radioactive beam induced reactions.

The author wishes to thank Z. Kohley and J. J. Kolata for sharing their data. Research sponsored by the Office of Nuclear Physics, U.S. Department of Energy.

\section{References}

1. W. Loveland, Phys. Rev. C 76, (2007) 014612

2. M. Dasgupta et al., Annu. Rev. Nucl. Part. Sci 48 (1998) 401

3. H. Timmers et al., Nucl. Phys. A 633, (1998) 421

4. F. Scarlassara et al., Nucl. Phys. A 672, (2000) 99

5. A. M. Stefanini et al., Phys. Rev. C 76, (2007) 014610

6. H. Q. Zhang et al., Phys. Rev. C 82, (2010) 054609 
7. D. Shapira et al., Nucl. Instrum. Methods Phys. Res. A 551, (2005) 330

8. J. F. Liang et al., Phys. Rev. C 75, (2007) 054607

9. K. T. Lesko et al., Phys. Rev. C 34, (1986) 2155

10. M. Dasgupta et al., Nucl. Phys. A 539, (1992) 351

11. K. Hagino et al., Comput. Phys. Commun. 123 (1999) 143

12. S. Raman et al., At. Data Nucl. Data Tables 36 (1987) 1

13. R. H. Spear, At. Data Nucl. Data Tables 42 (1989) 55

14. R. A. Broglia and A. Winther, Heavy Ion Reactions (Addison-Wesley, 1991) 114

15. N. Rowley and K. Hagino, Nucl. Phys. A 834 (2010) 110c

16. A. A. Sonzogni et al., Phys. Rev. C 57 (1998) 722

17. J. F. Liang, Nucl. Phys. A 834, (2010) 123c

18. C. -C. Sahm et al., Nucl. Phys. A 441, (1985) 316

19. A. C. Berriman et al., Nature 413, (2001) 144

20. A. M. Stefanini et al., Eur. Phys. J. 23, (2005) 473 